\title{
Pharmacological effects of Salvia miltiorrhiza (Danshen) on cerebral infarction
}

\author{
Tsai-Hui Lin and Ching-Liang Hsieh*1,2,3
}

\begin{abstract}
Danshen, the dried root of Salvia miltiorrhiza, is a Chinese medicine used to promote blood flow and treat vascular disease. The present article reviews the pharmacological effects of Danshen on cerebral infarction and possible interactions between Danshen and Western drugs. Danshen may reduce or prolong the development of atherosclerosis and may have anti-hypertensive and anti-platelet aggregation effects, which prevent cerebral infarction. Danshen may enhance endogenous anti-oxidative enzyme activities such as the expression of endothelial nitric oxide synthase and may scavenge oxygen free radicals. Prevention and treatment of cerebral infarction by Danshen involves multiple pathways, including anti-atherosclerosis, anti-hypertension, anti-platelet aggregation, antiinflammatory and anti-oxidative effects.
\end{abstract}

\section{Review}

\section{Background}

Danshen, the dried root of Salvia miltiorrhiza, is used in Chinese medicine to treat vascular disease. According to Chinese medicine theory, Danshen promotes blood flow and resolves blood stasis. Among stroke patients, $80 \%$ suffer from cerebral infarction and $20 \%$ cerebral hemorrhage [1]. Cerebral infarction is an ischemic condition of the brain including thrombosis, embolism or systemic hemodynamic hypotension. Cerebral infarction is often caused by atherosclerosis of large and small arteries. The etiology of atherosclerosis and stroke is related to inflammation and genetic factors. Ischemic cerebral infarction may be prevented through anti-inflammation and treatment for vascular diseases, heart diseases and hypertension [2-4]. Anti-thrombosis and thrombolysis are used to treat ischemic cerebral infarction [2]. The most effective method to re-establish cerebral blood flow is thrombolytic therapy; however, this therapy is often at the risk of bleeding [2]. Neuroprotection is also a potential treatment [5].

While Danshen or its ingredients reduce the infarction volume, the action mechanism of Danshen remains obscure [6-8]. Danshen contains the lipid-soluble tanshinone I (Tan I), tanshinone IIA (Tan IIA), cryptotanshi-

\footnotetext{
* Correspondence: clhsieh@mail.cmuh.org.tw

1 Department of Chinese Medicine, China Medical University Hospital, Taichung, 40402, Taiwan

Full list of author information is available at the end of the article
}

none and dihydrotan-shinone as well as the water-soluble danshensu and salvianolic acid B (Sal B) [9]. Danshen inhibits platelet aggregation and promotes fibrinolysis $[10,11]$.

Covering literature between 1990 and 2009, this article reviews basic and clinical studies on Danshen in the prevention and treatment for cerebral infarction. The key words used for search in the PubMed library were 'Danshen' or 'Salvia miltiorrhiza' combined with 'cerebral infarction', 'ischemic' or 'cerebral ischemia'.

\section{Prevention of cerebral infarction \\ Anti-atherosclerosis and anti-inflammatory effects}

Atherosclerosis is caused by endothelial damage, which may lead to platelet aggregation and the release of platelet factor, resulting in the proliferation of smooth muscle in the arterial intima. The process of atherosclerosis comprises inflammation and involves both innate and adaptive immunity [12]. The tanshinones components of Danshen (tanshinone I, dihydrotanshinone and cryptotanshinone) inhibit the production of interleukin-12 (IL12) induced by lipopolysaccharide (LPS)-activated macrophages and the production of interferon- $\gamma$ induced by keyhole limpet hemocyanin-primed lymph node cells [13]. Tanshinones also inhibit the expression of the IL-12 $\mathrm{p} 40$ gene and prevent nuclear factor $-\kappa B(\mathrm{NF}-\kappa \mathrm{B})$ from binding to $\kappa B$ site [13]. As IL-12 and NF- $\kappa B$ are closely associated with inflammatory responses, tanshinones may have anti-inflammatory effects [13]. Tumor necrosis 
factor- $\alpha$ (TNF- $\alpha)$, a pro-inflammatory cytokine, is regulated by NF- $\mathrm{KB}$ while vascular adhesion molecule-1 (VCAM-1) regulates the migration of leukocytes into the vessel wall. Pre-treatment with either aqueous ethanolic extract of Danshen or Sal B component of Danshen inhibits TNF- $\alpha$-induced expression of VCAM- 1 and TNF- $\alpha$ induced activation of NF- $\mathrm{KB}$ in human aortic endothelial cells, suggesting that both SME and Sal B posses antiinflammatory properties and are closely associated with atherogenesis because leukocytes migrate into the vessel wall in the early stage of atherogenesis [14]. Aqueous extract of Danshen suppresses the adhesion rate of neutrophils stimulated by TNF- $\alpha$, and also inhibits adhesion of neutrophils stimulated by $N$-formyl-methionyl-leucylphenylalanine (fMLP) [15]. Aqueous extract of Danshen inhibits TNF- $\alpha$-induced up-regulation of E-selectin, intracellular molecule-1 (ICAM-1) and VCAM-1 expression [15]. Danshen treatment lowers plasma viscosity, erythrocyte aggregation and fibrinogen levels in rats with traumatic brain injury [16]. Tan IIA increases estrogen receptor activity in HeLa cells. Tan IIA inhibits iNOS (inducible nitric oxide synthase) protein production and nitric oxide (NO) production and inhibits pro-inflammatory cytokine IL-1 $\beta$, IL- 6 and TNF- $\alpha$ via estrogen receptors in lipopolysaccharide (LPS) activated RAW 264.7 macrophages, suggesting that Tan IIA may serve as an estrogen-receptor-like modulator to produce immune responses [17]. C-reactive protein (CRP), an inflammation marker, induces pro-inflammatory cytokines through NF- $\mathrm{BB}$ and contributes to atherosclerosis in endothelial cells $[18,19]$. Tan IIA inhibits NF-kB-DNA complex, NF- $\mathrm{kB}$ binding activity and the phosphorylation of IKB $\alpha$ and inhibit the translocation of NF- $\mathrm{KB}$ from cytosol to nuclei, demonstrating that tanshinone possesses anti-inflammatory properties [20]. Tan IIA down-regulates protein expression and activities of matrix metalloproteinase-2 and -9 (MMP-2, MMP-9) and reduces the VCAM- 1 and IL- $1 \beta$ levels to suppress the increase in the aorta intimal area in rabbits treated with a high-fat-diet [21]. Triterpenoids-enriched extract of Danshen reduces the aortic atherosclerotic lesion area and inhibits inflammatory serum marker of CRP and monocyte chemotactic protein (MCP-1) [22]. Moreover, triterpenoids-enriched extract of Danshen reduces the serum levels of total cholesterol and triglyceride in low density lipoprotein receptor (LDLR) + mice via the inhibition of the inflammatory markers CRP and MCP-1 [22]. Triterpenoids are antiatherogenic which may be associated with its anti-inflammatory properties [22]. In summary, the anti-atherosclerosis and anti-inflammatory actions of Danshen play roles in the prevention of cerebral infarction.

\section{Anti-hypertensive effects}

Hypertension is a risk factor for cerebral infarction. Maintaining blood pressure within the ideal range has recently been recognized as an important principle in the prevention and treatment of ischemic cerebral infarction. Aqueous extract of Danshen alleviates hypertension in the two-kidney/one-clip (2K1C) Goldblatt renovascular hypertensive rats model and reduced the activity of serum angiotensin converting enzyme and the levels of serum aldosterone [23,24]. MTB70 is composed of 70\% $(70 \mathrm{mg} / \mathrm{ml}$ ) of Magnesium tanshinoate B (MTB) which is one of the aqueous active components of Danshen. MTB70 (0.7-175 mg/kg) reduces blood pressure more than the aqueous extract of Danshen with same volume in a saline- or phenylephrine $(2.5 \mu \mathrm{g} / \mathrm{kg}$ bolus injection and followed by $100 \mu \mathrm{g} / \mathrm{kg}$ at $0.13 \mathrm{ml} / \mathrm{hr}$ injection continuously) -induced hypertension model in rats; However this rapid and potent anti-hypertensive effect is short-lived [25]. Tanshinone IIA reduces blood pressure in the 2K1C renovascular hypertension model of hamsters [26]. Overall, the anti-hypertensive effects of Danshen include inhibition of angiotensin converting enzyme and generation of eNOS and/or vasodilatation.

\section{Treatment of cerebral infarction Anti-platelet aggregation effects}

Anti-platelet aggregation, which involves platelet adhesion, is widely used to treat acute ischemic cerebral infarction [27]. Platelet adhesion increases in mice with middle cerebral artery occlusion (MCAo) [28]. Eight derivatives of Danshen inhibit platelet aggregation induced by adenosine diphosphate (ADP) in vitro in rabbit plasma [29]. Salvianolic acid B (SAB) inhibits platelet deposition to collagen at venous and arterial shear rates, suggesting that $\mathrm{SAB}$ inhibits platelet adhesion to collagen via interfering collagen receptor $\alpha 2 \beta 1$ [30]. Salvianolic acid inhibits ADP-induced platelet aggregation in platelet-rich plasma and in washed platelets both in vivo and in vitro, probably through changing protein expression [31]. Danshen decreases the malondialdehyde (MDA) levels of platelets and increases the superoxide dismutase (SOD) activity of platelets to inhibit platelet aggregation in pulmonary thromboembolism induced by collagen and adrenaline in mice [32]. The 764-3 $(100 \mu \mathrm{g} / \mathrm{ml})$, purified compound of Danshen extract, inhibits platelet aggregation induced by arachidonic acid or ADP in humans and rabbits [33]. Danshen decreases the concentration of intra-platelet free calcium, which is closely associated with platelet aggregation and release [34]. Overall, Danshen exhibits anti-platelet aggregation effects via multiple pathways, e.g. the inhibition of intra-platelet calcium and anti-oxidant activities.

\section{Neuroprotection through anti-inflammatory effects}

Inflammatory responses are critically important to a patient after brain ischemia/reperfusion injury. The proinflammatory cytokines IL-1 $\beta$, TNF- $\alpha$ and IL- 6 increase after an ischemia attack, which enhances the expression 
of adhesion molecules including ICAM-1 and P-selectin, leading to brain edema and neuronal death $[35,36]$. In addition, leukocytes, neurons and activated microglial cells in ischemic damaged region release cytokines, chemokines and oxygen free radicals which lead to secondary brain tissue damage. Matrix metalloproteinases, which cleave protein components of the extracellular matrix, play a role in neurovascular remodeling and neuronal death $[35,36]$. The microglia may be activated during brain damage including ischemia, inflammation and infection [37]. The increases of activated microglia may represent the severity of neuronal damage in a MCAo rat model [38]. Danshen inhibits superoxide generation by microglia in primary microglia cell cultures from rat brains [39]. Danshen reduces CD18 and CD11b immunoreactive cells in the peri-vascular region and inhibits leukocyte infiltration and neuronal death in the cerebral infarction region in the ischemia-reperfusion MCAo rat model. As leukocytes are mediated via the combination of surface receptors of CD18 and CD11b and intracellular adhesion molecules of endothelium, the neuroprotection capability of Danshen may be associated with the inhibition of leukocyte adherence to the endothelium [40]. A study on the effects of Danshen on inflammatory response in cerebral ischemia and reperfusion injury in the MCAo rat model indicates that pre-treatment with Tanshinone IIA reduces the cerebral infarction area, neurological deficit score and cerebral edema. As pre-treatment with Tanshinone IIA reduces TNF- $\alpha$, the myeloperoxidase (MPO) marker of leukocytes, E-selectin and ICAM-1 in ischemic brain tissue and serum IL-8, Danshen may inhibit inflammatory responses to reduce brain damage induced by ischemia-reperfusion injury in rats [41]. The Danshen dripping pill (DDP) is composed of Danshen, Panax notoginseng and Dryobalanops camphor. A controlled pilot study (106 patients) found that the recurrent rate in the DDP group was $9.6 \%$ compared with $24.1 \%$ in the non-DDP group. The serum CRP levels decreased from 2.33 to $1.50 \mathrm{mg} / \mathrm{L}$ in the DPP group were greater than those decreased from 2.21 to $1.77 \mathrm{mg} / \mathrm{L}$ in the non-DDP group [42]. Overall, Danshen has antiinflammatory activity and may improve cerebral infarction and provide neuroprotection.

\section{Neuroprotection through anti-oxidative effects}

Nitrogen and oxygen free radicals involved in the production of reactive oxygen species (ROS) play an important role in brain damage during the reperfusion period following ischemia $[43,44]$. These ROS, which are involved in brain damage after cerebral ischemia, affect the signal transmission of mitochondria and DNA repair of enzyme and transcription factors, leading to apoptosis [45]. Danshensu and salvianolic acid B are phenolic acids of Danshen, both of which have scavenging activities towards free radicals of hydroxyl, 1,1-diphenyl-2-picryl-hydrazyl
(DPPH), 2-azino-bis(3-ehtylbenzthiazoline-6-sulfonic acid) (ABTS) free radicals, hydrogen peroxidase and superoxide anion in human umbilical vein endothelial cells [46]. Our previous studies show that Danshen reduces the cerebral infarction volume and the neurological deficit score and reduces luminal-chemiluminescence counts [8]. As luminal-chemiluminescence counts may represent ROS, these effects of Danshen may be associated with its free radical scavenging activities in ischemia-reperfusion injured rats [8]. Intra-abdominal administration of Tan IIB, a major active component of Danshen, reduces the focal infarct volume, neurological deficit and apoptosis in the MCAo rat model. As apoptosis is a major pathway leading to neuronal death, Tan IIB is neuroprotective [47]. Damage from oxygen free radicals occurs in the ischemic/reperfusion injury under oxidation response to cell membrane lipids, proteins or DNA. Inhibiting oxidation and free radical scavenging are important in the treatment of ischemic cerebral infarction. A study using a 4-vessel occlusion rat model $[48,49]$ found that (1) Danshen reduced an increase of cerebral NO and MDA 30 minutes prior to occlusion and that (2) pre-treatment with Danshen increased a significantly decreased SOD activity. NO mediates glutamate neurotoxicity and the inhibition of NO synthase may prevent the development of brain edema. SOD is a scavenger of superoxide anion and the levels of MDA may reflect the degree of lipid peroxidation. Thus Danshen is neuroprotective in this occlusion and reperfusion rat model $[48,49]$. Isopropyl- $\beta,-(3,4-$ dihydroxyphenyl)- $\alpha$-hydroxypropanoate (ND-309), a metabolite of Danshen in rat brain, reduces cerebral infarction volume, brain edema and neurological deficit in the MCAo rat model. Moreover, ND309 reduces brain tissue MDA and increases the reduction of ATP induced by ischemia-reperfusion injury [50] of mitochondrial ATP, mitochondrial SOD and glutathione peroxidase (GSH-Px) activities induced by ischemia-reperfusion injury. ND-309 is neuroprotective role to reduce brain damage induced by cerebral ischemia [50]. Danshen reduces the neurological deficit score and the levels of MDA and increases SOD activities in the MCAo rat model [51]. Pretreatment with Danshen may reduce brain edema and MDA concentration of cerebral cortex and hippocampus region in an ischemia-reperfusion injured rat model while Danshen may increase levels of catalase, SOD, GSH and ATP of the cerebral cortex and hippocampus region [52]. In summary, Danshen demonstrates neuroprotective effects that are closely associated with anti-oxidative action.

\section{Drug interactions with warfarin}

Warfarin is an anticoagulant used to prevent atrial fibrillation, valvular heart disease, ischemic stroke and deep venous thrombosis [53]. Danshen inhibits platelet adhe- 
sion and aggregation and suppresses the formation of thromboxane $\mathrm{A}_{2}$ [53]. The interaction between Danshen and warfarin can cause bleeding and prolong prothrombin time or the International Normalized Ratio (INR) [53]. Co-administration of Danshen and warfarin should be avoided or closely monitored [54]. Tanshinones inhibits CYP1A1, CYP2C6 and CYP2C11-mediated warfarin metabolism to increase the concentration of warfarin [55]. Aspirin prevents and alleviates cerebral infarction. Danshen displaces the binding between aspirin and protein, thereby increasing free aspirin concentration in serum [56]. Atrial fibrillation, which may be prevented and treated by digoxin, is closely related to cerebral infarction induced by embolus. Danshen has digoxin-like immunoreactivity leading to a false interference of digoxin concentration [56].

\section{Compared free radical scavenging ability with other herbs}

The extracts of Ginkgo biloba L. leaves (Egb 761), with free radical scavenging activity, reduce the size of cerebral infarction and improve neurological behavior in rats with permanent and transient MCAo [57,58]. Egb 761 is widely used to treat ischemic cerebral infarction. The concentration of salvianolic acid B is lower than Egb 761 in scavenging activity of superoxide anion and hydroxyl radicals in rat microsome, and in $\mathrm{SH}-\mathrm{SY} 5 \mathrm{Y}$ induced by $\mathrm{H}$ ${ }_{2} \mathrm{O}_{2}$ [59]. Panax notoginseng (Sanqi) is also used to treat vascular disorders. Danshen is stronger than Panax notoginseng in scavenging activity of superoxide anion, hydroxyl and DPPH radicals, yet it is weaker than Panax notoginseng in the scavenging activity of hydrogen peroxide and ferrous ion chelating activity [60]. While Sanqi demonstrates strong ferrous ion chelating activity and strong scavenging activities of hydrogen peroxide and hydroxyl radicals, it is weak in the scavenging activities of superoxide anion and DPPH radicals. Therefore, the scavenging capabilities of Danshen and Sanqi are quite different among the various free radicals [60].

\section{Conclusion}

Prevention and treatment of cerebral infarction by Danshen involves multiple pathways, including anti-atherosclerosis, anti-hypertension, anti-platelet aggregation, anti-inflammatory and anti-oxidative effects (Additional file 1).

\section{Additional material}

Additional file 1 Possible pharmacological actions of Danshen for prevention and treatment of cerebral infarction. Supplemental table

\section{Abbreviations}

Tan I: tanshinone I; Tan II: tanshinone II; Sal B: salvianolic acid B; LPS: lipopolysaccharide; NF-kB: nuclear factor-kB; TNF-a: tumor necrosis factor-a; VCAM-1: vascular adhesion molecule-1; fMLP: N-formyl-methionyl-leucyl-phenylalanine;
ICAM-1: intracellular molecule-1; iNOS: inducible nitric oxide synthase; NO: nitric oxide; MMP-2, MMP-9: matrix metalloproteinase-2 and -9; MCP-1: monocyte chemotactic protein; LDLR: low density lipoprotein receptor; 2K1C: twokidney/one-clip; SME: Salvia miltiorrhiza extract; MTB: magnesium tanshinoate B; MCAo: middle cerebral artery occlusion; ADP: adenosine diphosphate; MDA: malondialdehyde; SOD: superoxide dismutase; MPO: myeloperoxidase; DDP: Danshen dripping pill; ROS: reactive oxygen species; DPPH: 1,1-diphenyl-2-picryl-hydrazyl; ABTS: 2-azino-bis(3-ehtylbenzthiazoline-6-sulfonic acid); ND-309: isopropyl- $\beta$,-(3,4-dihydroxyphenyl)-a-hydroxypropanoate; Egb 761: the extracts of Ginkgo biloba L. leaves

\section{Competing interests}

The authors declare that they have no competing interests.

\section{Authors' contributions}

THL searched the literature, organized the data and wrote the manuscript. CLH analyzed the data and revised the manuscript. Both authors read and approved the final version of the manuscript.

\section{Acknowledgements}

We thank the editorial team of the journal of Chinese Medicine for their valuable comments and editorial assistance. This study was supported in part by the Department of Health Clinical Trial and Research Center of Excellence, Taiwan (DOH99-TD-B-111-004).

\section{Author Details}

'Department of Chinese Medicine, China Medical University Hospital, Taichung, 40402, Taiwan, ${ }^{2}$ Graduate Institute of Acupuncture Science, China Medical University, Taichung, 40402, Taiwan and ${ }^{3}$ Acupuncture Research Center, China Medical University, Taichung, 40402, Taiwan

Received: 17 February 2010 Accepted: 21 June 2010

Published: 21 June 2010

\section{References}

1. Dietl M, Pohle R, Weingärtner M, Polgar R, Grässel E, Schwab S, Kolominsky-Rabas P: Stroke etiology and long-term need of care in ischemic stroke patients. Fortschr Neurol Psychiatr 2009, 77(12):714-719.

2. Albers GW, Amarenco P, Easton JD, Sacco RL, Teal P: Antithrombotic and thrombolytic therapy for ischemic stroke: American college of chest physicians evidence-based clinical practice guidelines. Chest 8th edition. 2008, 133(6):630S-669S.

3. Mostaza JM, Martín-Jadraque R, Vicente I, San Martin MA, Lahoz C. Patients at high risk of cerebrovascular disease: the REACH study. Cerebrovasc Dis 2009, 27(1):77-81.

4. Liu LS, Caguioa ES, Park CG, Quek DKL, Saito I, Venketasubramanian N, Wong KSL, Reid JL: Reducing stroke risk in hypertensive patients: Asian Consensus Conference recommendations. Int J Stroke 2006 1(3):150-157.

5. Tuttolomondo A, Di Sciacca R, Di Raimondo D, Arnao V, Renda C, Pinto A, Licata G: Neuron protection as a therapeutic target in acute ischemic stroke. Curr Top Med Chem 2009, 9(14):1317-1334.

6. Kuang $P$, Wu W, Zhu K: Evidence for amelioration of cellular damage in ischemic rat brain by radix salviae miltiorrhizae treatmentimmunocytochemistry and histopathology studies. J Tradit Chin Med 1993, 13(1):38-41. In Chinese

7. Wu W, Kuang P, Li Z: Protective effect of radix Salviae miltiorrhizae on apoptosis of neurons during focal cerebral ischemia and reperfusion injury. J Tradit Chin Med 1997, 17(3):220-5. In Chinese

8. Lao CJ, Lin JG, Kuo JS, Chiang SY, Chen SC, Liao ET, Hsieh CL: Effects of Salvia Miltiorrhiza Bunge on cerebral infarct in ischemia-reperfusion injured rats. Am J Chin Med 2003, 31(2):191-200.

9. Adams JD, Wang R, Yang J, Lien EJ: Preclinical and clinical examinations of Salvia miltiorrhiza and its tanshinones in ischemic conditions. Chin Med 2006, 1:3

10. Zhou L, Zuo Z, Chow MSS: Danshen: an overview of its chemistry, pharmacology, pharmacokinetics, and clinical use. J Clin Pharmacol 2005, 45(12):1345-59.

11. Chan TY: Interaction between warfarin and danshen (Salvia miltiorrhiza). Ann Pharmacother 2001, 35(4):501-504. 
12. Libby P, Ridker PM, Hansson GK: Inflammation in atherosclerosis: from pathophysiology to practice. J Am Coll Cardiol 2009, 54(23):2129-2138

13. Kang BY, Chung SW, Kim SH, Ryu SY, Kim TS: Inhibition of interleukin-12 and interferon-gamma production in immune cells by tanshinones from Salvia miltiorrhiza. Immunopharmacology 2000, 49(3):355-61.

14. Chen YH, Lin SJ, Ku HH, Shiao MS, Lin FY, Chen JW, Chen YL: Salvianolic acid $B$ attenuates VCAM-1 and ICAM-1 expression in TNF-alpha-treated human aortic endothelial cells. J Cell Biochem 2001, 82(3):512-21.

15. Ren DC, Du GH, Zhang JT: Inhibitory effect of the water-soluble extract of Salvia miltiorrhiza on neutrophil-endothelial adhesion. Jpn J Pharmacol 2002, 90(3):276-80

16. Zhang J, Yi S, Zhang X: Effects of salvia miltiorrhiza on hemorheological properties after brain injury. Chin J Neurosurg 1995, 11(1):784-790. In Chinese

17. Fan GW, Gao XM, Wang H, Zhu Y, Zhang J, Hu LM, Su YF, Kang LY, Zhang BL: The anti-inflammatory activities of Tanshinone IIA, an active component of TCM, are mediated by estrogen receptor activation and inhibition of iNOS. J Steroid Biochem Mol Biol 2009, 113(3-5):275-280.

18. Verma S, Badiwala MV, Weisel RD, Li SH, Wang CH, Fedak PWM, Li RK, Mickle DAG: C-reactive protein activates the nuclear factor-kappaB signal transduction pathway in saphenous vein endothelial cells: implications for atherosclerosis and restenosis. JThorac Cardiovasc Surg 2003, 126(6):1886-1891.

19. Liang YJ, Shyu KG, Wang BW, Lai LP: C-reactive protein activates the nuclear factor-kappaB pathway and induces vascular cell adhesion molecule-1 expression through CD32 in human umbilical vein endothelial cells and aortic endothelial cells. J Mol Cell Cardiol 2006, 40(3):412-420

20. Jang SI, Kim HJ, Kim YJ, Jeong SI, You YO: Tanshinone IIA inhibits LPSinduced NF-kappaB activation in RAW 264.7 cells: possible involvement of the NIK-IKK, ERK1/2, p38 and JNK pathways. Eur $J$ Pharmacol 2006, 542(1-3):1-7.

21. Fang ZY, Lin R, Yuan BX, Liu Y, Zhang H: Tanshinone IIA inhibits atherosclerotic plaque formation by down-regulating MMP- 2 and MMP-9 expression in rabbits fed a high-fat diet. Life Sci 2007, 81(1718):1339-1345.

22. Zhang Q, Chang Z, Yang J, Wang Q: Antiatherogenic property of triterpenoids-enriched extract from the aerial parts of Salvia miltiorrhiza. Phytother Res 2008, 22(8):1040-1045.

23. Kang DG, Yun YG, Ryoo JH, Lee HS: Anti-hypertensive effect of water extract of danshen on renovascular hypertension through inhibition of the renin angiotensin system. Am J Chin Med 2002, 30(1):87-93.

24. Tian S, Yu ZY, Tao F: Effects of Salvia miltiorrhizae renal intracasular injection to local angiotensin II in one-kidney, one clip hypertension rats. Chin J Integr Med 1996, 16:94-96. In Chinese

25. Leung SWS, Zhu DY, Man RYK: Effects of the aqueous extract of Salvia Miltiorrhiza (danshen) and its magnesium tanshinoate B-enriched form on blood pressure. Phytother Res 2009, 24(5):769-774.

26. Kim DD, Sánchez FA, Durán RG, Kanetaka T, Durán WN: Endothelial nitric oxide synthase is a molecular vascular target for the Chinese herb Danshen in hypertension. Am J Physiol Heart Circ Physiol 2007, 292(5):H2131-2137

27. Bednar MM, Gross CE: Antiplatelet therapy in acute cerebral ischemia. Stroke 1999, 30:887-893.

28. Terao S, Yilmaz G, Stokes KY, Russell J, Ishikawa M, Kawase T, Granger DN: Blood cell-derived RANTES mediates cerebral microvascular dysfunction, inflammation, and tissue injury after focal ischemiareperfusion. Stroke 2008, 39:2560-70.

29. Jin X, Chen BL, Wu WJ, Gu Z, Jiang MH, Zhang ZH, Dong JC: Effects of eight danshensu's derivatives on rabbit platelet aggregation. $J$ Shanghai Med Univ 2000, 27(3):181-184. In Chinese

30. Wu YP, Zhao XM, Pan SD, Guo DA, Wei R, Han JJ, Kainoh M, Xia ZL, de Groot PG, Lisman T: Salvianolic acid B inhibits platelet adhesion under conditions of flow by a mechanism involving the collagen receptor alpha2beta1. Thromb Res 2008, 123(2):298-305.

31. Yao Y, Liu AH, Wu WY, Guan SH, Jiang BH, Yang M, Bi KS, Liu X, Guo DA: Possible target-related proteins of salvianolic acids in rat platelets. Phytochem Lett 2008, 1:135-138.

32. Wang $X$, Pang J, Shan $C$, Yang S, Zheng Y: Effect of danshen injection on pulmonary thromboembolism and platelet free radical levels in mice. Zhongguo Zhong Yao Za Zhi 1996, 21(9):558-560. In Chinese
33. Wang Z, Zhang $\mathrm{H}$, You H: Effect of danshen injection on pulmonary thromboembolism and platelet free radical levels in mice. Zhongguo $Y_{i}$ Xue Ke Xue Yuan Xue Bao 1994, 16(2):140-143. In Chinese

34. Zhong $\mathrm{P}, \mathrm{Lin} X$ : Effect of danshen injection on cytosolic free calcium concentration in normal rat platelet. Chin J Integr Surg 2005 11(2):141-142. In Chinese

35. Amantea D, Nappi G, Bernardi G, Bagetta G, Corasaniti MT: Post-ischemic brain damage: pathophysiology and role of inflammatory mediators. FEBS J 2009, 276(1):13-26

36. Xia W, Han J, Huang G, Ying W: Inflammation in ischemic brain injury: current advances and future perspectives. Clin Exp Pharmacol Physiol 2009, 37(2):253-258

37. Korematsu K, Goto S, Nagahiro S, Ushio Y: Microglial response to transient focal cerebral ischemia: an immunochemical study on the rat cerebral cortex using anti-phosphotysine antibody. J Cereb Blood Flow Metab 1994, 14:825-830

38. Morioka T, Kalehua AN, Streit WJ: The microglial reaction in the rat dorsal hippocampus following transient forebrain. I Cereb Blood Flow Metab 1991, 11:966-973.

39. Koo BS, Kwon TS, Kim CH: Salviae miltiorrhizae radix inhibits superoxide generation by activated rat microglia and mimics the action of amphetamine on in vitro rat striatal dopamine release. Neruochem Res 2004, 29(10):1837-1845

40. Liu J, Kuang P, Li B, Wu W, Zhang F: Experiment studies on the mechanism of radix Salviae miltiorrhizae (RSM) induced neuroprection-reperfusion rats. Chin J Neuroimmol \& Neurol 1998, 5(2):77-82. In Chinese

41. Hu XM, Zhou MM, Hu XM, Wang J, Zeng FD: The effects of sodium $\beta$ aescinate on inflammatory process induced by focal cerebral ischemiareperfusion in rats. Chin Pharmacol Bull 2006, 22(4):436-440. In Chinese

42. Xu G, Zhao W, Zhou Z, Zhang R, Zhu W, Liu X: Danshen extracts decrease blood $C$ reactive protein and prevent ischemic stroke recurrence: a controlled pilot study. Phytother Res 2009, 23(12):1721-1725.

43. Love S: Oxidative stress in brain ischemia. Brain Pathol 1999, 9(1):119-131.

44. Gursoy-Ozdemir Y, Can A, Dalkara T: Reperfusion -induced oxidative/ nitrative injury to neurovascular unit after focal cerebral ischemia. Stroke 2004, 35:1449-1453.

45. Chan $\mathrm{PH}$ : Reactive oxygen radicals in signaling and damage in the ischemic brain. J Cereb Blood Flow Metab 2001, 21:2-14.

46. Zhao GR, Zhang HM, Ye TX, Xiang ZJ, Yuan YJ, Guo ZX, Zhao LB: Characterization of the radical scavenging and anti-oxidant activities of danshensu and salvianolic acid B. Food Chem Toxicol 2008, 46:71-81

47. Yu XY, Lin SG, Zhou ZW, Chen X, Liang J, Duan W, Yu XQ, Wen JY, Chowbay B, Li CG, Sheu FS, Chan E, Zhou SF: Tanshinone IIB, a primary active constituent from Salvia miltiorrhza, exhibits neuro-protective activity in experimentally stroked rats. Neurosci Lett 2007, 417(3):261-265.

48. Kuang $P$, Tao $Y$, Tian $Y$ : Radix Salviae miltiorrhizae treatment results in decreased lipid peroxidation in reperfusion injury. J Tradit Chin Med 1996, 16(2):138-142. In Chinese

49. Kuang $P$, Tao $Y$, Tian $Y$ : Effect of radix Salviae miltiorrhizae on nitric oxide in cerebral ischemic-reperfusion injury. J Tradit Chin Med 1996, 16(3):224-227. In Chinese

50. Tian J, Li G, Liu Z, Zhang S, Qu G, Jiang W, Fu F: ND-309, a novel compound, ameliorates cerebral infarction in rats by antioxidant action. Neurosci Lett 2008, 442(3):279-283.

51. Liu C, Min LQ, Ji ZS, W Q, Jia YJ, Li SY: Protective effects of Salvia miltiorrhizae on oxidative stress in rats with focal cerebral ischemia. Chin J Clin Rehabil 2006, 10(3):37-39. In Chinese

52. Mo Z, Zheng Y, Pan Z, Liang R: Protective effect and mechanism of salvia miltiorrhiza on cerebral ischemia reperfusion injury in rats. Chin Herb Pharmacol Clin 1998, 14(4):24-26. In Chinese

53. Cheng TO: Danshen: what every cardiologist should know about this Chinese herbal drug. Int J Cardiol 2006, 110:411-412.

54. Holbrook AM, Pereira JA, Labiris R, McDonald H, Douketis JD, Crowther M, Wells PS: Systemic overview of warfarin and its drug and food interactions. Arch Intern Med 2005, 165:1095-1106.

55. Wu WWP, Yeung JHK: Inhibition of warfarin hydroxylation by major tanshinones of Danshen (Salvia miltiorrhiza) in the rat in vitro and in vivo. Phytomedicine 2009, 17(3-4):219-226.

56. Cheng TO: Cardiovascular effectsof danshen. Int J Cardio/ 2007 121:9-12. 
57. Ellnain-Wojtaszek M, Kruczyński Z, Kasprak J: Investigation of the free radical scavenging activity of Ginkgo biloba L. leaves. Fitoterapia 2003, 74:1-6.

58. Lee EJ, Chen HY, Wu TS, Chen TY, Ayoub IA, Kenneth I, Maynard MJ: Acute administration of Ginkgo biloba extract (EGb761) affords neuroprotection against permanent and transient focal cerebral ischemia in Spragrue-Dawley rats. J Neurosci Res 2002, 68:636-645.

59. CS Liu, Cheng Y, Hu JF, Zhang W, Chen NH, Zhang JT: Comparison of antioxidant activities between salvianolic acid $B$ and Ginkgo biloba extract (EGb 761). Acta Pharmacol Sin 2006, 27(9):1137-1145.

60. Zhao GR, Xiang ZJ, Ye TX, Yuan YJ, Guo ZX: Antioxidant activities of Salvia miltiorrhiza and Panax notoginseng. Food Chem 2006, 99:767-774.

doi: $10.1186 / 1749-8546-5-22$

Cite this article as: Lin and Hsieh, Pharmacological effects of Salvia miltiorrhiza (Danshen) on cerebral infarction Chinese Medicine 2010, 5:22

Submit your next manuscript to BioMed Central and take full advantage of:

- Convenient online submission

- Thorough peer review

- No space constraints or color figure charges

- Immediate publication on acceptance

- Inclusion in PubMed, CAS, Scopus and Google Scholar

- Research which is freely available for redistribution

Submit your manuscript at www.biomedcentral.com/submit
C) Biomed Central 